Editorial

\title{
Operating Room to Intensive Care Unit Handoffs: Time to Recognize an Underrated Predictor of Patient Outcome
}

\author{
Girija P. Rath ${ }^{1, \oplus}$ Gyaninder P. Singh ${ }^{1, \oplus}$ \\ ${ }^{1}$ Department of Neuroanaesthesiology and Critical Care, All India \\ Institute of Medical Sciences (AIIMS), New Delhi, India
}

J Neuroanaesthesiol Crit Care 2019;6:179-181

In patient care, the term "handoffs" or "handovers" refers to transferring the responsibility of patient's well-being between the individual providers and/or teams. It has been identified by the World Health Organization (WHO) as a part of "Action of patient safety: High 5 s initiatives." Such transition of care may occur at various stages from admission to discharge of patient from the hospital. Handoffs in surgical branches mainly focus on the perioperative interventions, whereas in medical specialties it would focus on the current condition of the patient. One of the very crucial handoffs take place in the postoperative period when the patient is transported with monitoring from the operating room (OR) to the postanesthesia care unit (PACU) or the intensive care unit (ICU). Practically, the transfer of patient care between the providers require passing of all the critical clinical information. Poor handoffs may lead to serious clinical consequences or mishaps. ${ }^{2}$ If the information is omitted or misunderstood there can be errors in clinical decision making, thereby causing potential harm to the patient., ${ }^{3,4}$ Patients are at the risk of adverse events during transfer of care and require a welldefined protocol for handoffs for improving safety. Many hospitals implement a checklist to provide a structured format for handoffs while transferring the responsibility of patient care between the providers. This helps to standardize the process and ensures that all relevant critical information is passed when patient care is transferred to a new team.

The goal of the handoff is to develop a shared understanding of the patient. An effective handoff should have four phases. ${ }^{5,6}$

- Prehandoff: The person who gives the handoffs must organize and update information in advance.

- On arrival: Time is fully devoted for the handoff to occur and other works should be stopped during that time.

- Dialogue: An exchange of information takes place between the giver and receiver. Ideally, this should be a verbal as well as written on patient chart or documented electronically.
- Posthandoff: The receiver of information integrates the new information and assumes care of the patient.

Elements of an effective handoff include both verbal and written communications. Verbal communication allows sharing of information including anticipated issues and tasks to be completed. It also allows active questioning and formation of "shared mental model." According to this concept, the team works more efficiently when there is shared understanding of the patient condition and the tasks to be performed. ${ }^{7}$ The written communication supplements the verbal communication and serves as a "transition record" for all relevant information including critical events; it may be paper based or electronic records.

Handoff is not just transfer of patient care information but also the transfer of patient responsibility. The strategies to improve handoff should include the following things:

- Standardization of the content and structure of both verbal and written communication helps in formation of standard protocols for handoff. ${ }^{5}$ This improves the consistency of information during handoff. Use of standardized protocols during handoff has shown to decrease the preventable adverse events. ${ }^{8,9}$ Also, simultaneous use of verbal and written handoffs resulted in more retention of the information compared with verbal handoff alone. ${ }^{10}$

- Verbal communication and interactive questioning: Faceto-face verbal communication and ability to actively question/interact is important for transfer of information and are recommended during handoff. Also, the use of standardized language during verbal communication ensures transfer of consistent information and proper understanding.

- Written templates for handoffs: Use of structured templates (checklists) for transfer of written information ensures consistency in the information relayed between the outgoing and incoming team and maintains same continuity of patient care between them.
Copyright $\odot 2019$ Indian Society of Neuroanaesthesiology and Critical Care

License terms

$10.1055 / \mathrm{s}-0039-3401790$

ISSN 2348-0548.
Girija P. Rath, MD, DM, Department

of Neuroanaesthesiology and Critical

Care, Neurosciences Centre, All

India Institute of Medical Sciences

(AlIMS), New Delhi 110029, India

(e-mail: girijarath@yahoo.co.in). 
- Minimizing interruptions at the time of handoff allows for the giver and receiver to focus on the exchange of information. This is a crucial step and adequate time should be given for the handoff to take place.

- Avoid "double handoffs," that is, when a care provider temporarily takes care of the patient for a short period and then transfers the care to another care provider. In such a situation, both the care providers during second handoff do not have primary knowledge of the patient.

- Communicate in a structured manner and focus on the important information to be passed on to the receiver at the time of handoff. It is important to emphasize on the tasks to be done and to give specific suggestions or recommendations for anticipated events that may occur. It is more likely to be remembered than general information passed.

- Use of read-back and interactive questioning ensures that the information has been adequately and effectively relayed between the giver and receiver. This would reduce error as well as prevent patient harm.

- Active listening behavior improves receipt of information during handoff and should be encouraged. This includes notetaking and questioning, listening, understanding, and processing of information by the recipients. Writing notes during handoff has been shown to reduce data loss, increase retention of information, and improve patient care delivery. ${ }^{10,11}$ Passive listening behavior, such as silent-listening or head nodding, does not confirm comprehension.

- The receiver must confirm successful comprehension of information and must ensure transfer of professional responsibility. ${ }^{12}$

There are certain important tasks and clinical information, communication of which should be prioritized by either team during the process of handoffs. ${ }^{13}$ They are as follows:

- Transfer of monitoring gadgets from OR to ICU and return back to OR as desired.

- Secure all the vascular accesses, devices, drainage tubes, catheters, and endotracheal tube.

- Identify ongoing medications and their dosages.

- Maintain adequate level of sedation and analgesia, cardiorespiratory, and hemodynamic stability.

In an effort to reduce handoff errors and increase patient safety many health organizations and committees have given recommendations for standardized approach to handoffs. ${ }^{4}$ The Association of American Medical Colleges (AAMC) has included handoffs on the list of competencies needed for residency. ${ }^{14}$ Similarly, Accreditation Council for Graduate Medical Education (ACGME) has made a program that includes transition of patient care or handoffs as one of the six focus areas while institutional teaching and clinical alignment, that is, Clinical Learning Environment Review (CLER) program. ${ }^{15,16}$ There are numerous interventions that aim to improve patient care by efficient handoffs, but standardized, reliable measurement tools are not widely used. A postoperative handover assessment tool (PoHAT) developed for assessing the handoff after surgery showed good feasibility and reliablility. ${ }^{17}$ Another tool to evaluate verbal handoff (Verbal Handoff Assessment Tool [VHAT]) demonstrated improved scores after the implementation of standardized handoffs protocols. In addition, there are tools developed to evaluate the quality of written handover documents, which have been tested in critical care settings. ${ }^{18}$ Different mnemonics are also utilized for the same purpose.

Neurosurgical procedure and the care provided during the perioperative period are more complex. Most of the patients require intensive care during immediate postoperative period; hence, several centers prefer to shift the postneurosurgical patients directly to the ICU from OR. However, the handoffs activities are vulnerable owing to a smaller ratio of resident doctors to the number of patients requiring handoffs. Moreover, there is lack of clear-cut policy in this context. In the Patient assessment, Assertive communication, Continuum of care, Teamwork with trust (PACT) Project, it was found that the handoffs among residents remain unstructured and important information on the patients are not communicated adequately..$^{19}$ Occurrence of handoffs by residents was observed to be an independent predictor of length of hospital and ICU stay, in neurosurgical patients. ${ }^{20}$ In a standardized system, which included brief operative/ anesthetic notes (written) and in-person encounters among residents and registered nurses, timely transfer of postoperative data was found to maximize safety and continuity of care in neurosurgical patients. ${ }^{21}$ However, we need to have more structured handoffs programs and their impact on patient outcome with randomized controlled trials, to reduce the burden of preventable medical errors in neurosurgical patient population.

\section{Conflict of Interest}

None declared.

\section{References}

1 World Health Organization. Patient safety. Action on patient safety: high 5s. Available at: https://www.who.int/ patientsafety/solutions/high5s/High5_overview.pdf. Accessed November 28, 2019

2 Sutcliffe KM, Lewton E, Rosenthal MM. Communication failures: an insidious contributor to medical mishaps. Acad Med 2004;79(2):186-194

3 Kripalani S, LeFevre F, Phillips CO, Williams MV, Basaviah P, Baker DW. Deficits in communication and information transfer between hospital-based and primary care physicians: implications for patient safety and continuity of care. JAMA 2007;297(8):831-841

4 Horwitz LI, Moin T, Krumholz HM, Wang L, Bradley EH. Consequences of inadequate sign-out for patient care. Arch Intern Med 2008;168(16):1755-1760

5 Arora V, Johnson J. A model for building a standardized handoff protocol. Jt Comm J Qual Patient Saf 2006;32(11):646-655

6 Gibson SC, Ham JJ, Apker J, Mallak LA, Johnson NA. Communication, communication, communication: the art of the handoff. Ann Emerg Med 2010;55(2):181-183

7 Cohen MD, Hilligoss B, Kajdacsy-Balla Amaral AC. A handoff is not a telegram: an understanding of the patient is co-constructed. Crit Care 2012;16(1):303

8 Starmer AJ, Spector ND, Srivastava R, Allen AD, Landrigan CP, Sectish TC; I-PASS Study Group. I-pass, a mnemonic to standardize verbal handoffs. Pediatrics 2012;129(2):201-204

9 Starmer AJ, Spector ND, Srivastava R, et al. I-PASS Study Group. Changes in medical errors after implementation of a handoff program. N Engl J Med 2014;371(19):1803-1812 
10 Bhabra G, Mackeith S, Monteiro P, Pothier DD. An experimental comparison of handover methods. Ann R Coll Surg Engl 2007;89(3):298-300

11 Pothier D, Monteiro P, Mooktiar M, Shaw A. Pilot study to show the loss of important data in nursing handover. $\mathrm{Br} J$ Nurs 2005; 14(20):1090-1093

12 Apker J, Mallak LA, Gibson SC. Communicating in the "gray zone": perceptions about emergency physician hospitalist handoffs and patient safety. Acad Emerg Med 2007;14(10):884-894

13 Wheeler DS, Sheets AM, Ryckman FC. Improving transitions of care between the operating room and intensive care unit. Transl Pediatr 2018;7(4):299-307

14 Englander R, Flynn T, Call S, et al. Toward defining the foundation of the MD degree: core entrustable professional activities for entering residency. Acad Med 2016;91(10):1352-1358

15 Nasca TJ, Day SH, Amis ES, Jr; ACGME Duty Hour Task Force. The new recommendations on duty hours from the ACGME Task Force. N Engl J Med 2010;363(2):e3

16 Weiss KB, Bagian JP, Wagner R, Nasca TJ. Introducing the CLER pathways to excellence: a new way of viewing clinical learning environments. J Grad Med Educ 2014;6(3):608-609
17 Nagpal K, Abboudi M, Fischler L, et al. Evaluation of postoperative handover using a tool to assess information transfer and teamwork. Ann Surg 2011;253(4):831-837

18 Nanchal R, Aebly B, Graves G, et al. Controlled trial to improve resident sign-out in a medical intensive care unit. BMJ Qual Saf 2017;26(12):987-992

19 Tapia NM, Fallon SC, Brandt ML, Scott BG, Suliburk JW. Assessment and standardization of resident handoff practices: PACT project. J Surg Res 2013;184(1):71-77

20 Kuhn EN, Davis MC, Agee BS, Oster RA, Markert JM. Effect of resident handoffs on length of hospital and intensive care unit stay in a neurosurgical population: a cohort study. J Neurosurg 2016;125(1):222-228

21 Birk HS, Han SJ, Rolston JD, et al. Resident-led implementation of a standardized handoff system to facilitate transfer of postoperative neurosurgical patients to the ICU. Cureus 2016;8(1):e461 